Medios digitales e inclusión social

Digital Media and Social Inclusion

\author{
Mark Warschauer \\ University of California, Irvine \\ markw@uci.edu \\ Melissa Niiya \\ University of California, Irvine \\ mniiya@uci.edu
}

Recibido: 23-4-2014

Aprobado: 22-5-2014 


\title{
Resumen
}

Mientras que las desigualdades vinculadas con el acceso a la tecnología y a su uso ocupan un lugar central en la política y la práctica educativa, tanto los educadores como los gestores de política no se ponen de acuerdo en cómo definir y conceptualizar la noción de «brecha digital». Esta brecha podría ser concebida como la falta de acceso a la tecnología y como un problema que se resuelve simplemente con proveer Internet y computadoras. En la medida en que el acceso a la tecnología básica ha aumentado, la atención se ha puesto más bien en las disparidades existentes en el rango de factores que permiten que la tecnología se use de modo efectivo. En lugar de un marco tecnocéntrico, que concibe el acceso a la tecnología en sí misma como la solución a la desigualdad, este artículo utiliza conceptualizaciones críticas de la literacidad para reconceptualizar la brecha digital en términos de inclusión social a través de la tecnología. El artículo provee ejemplos de iniciativas que buscan solucionar la brecha digital en India, Irlanda, Egipto y Perú. Sobre la base de estos ejemplos e investigación, este trabajo describe un marco para usar la tecnología para la inclusión social. Dicho marco busca reorientar la discusión de la brecha digital hacia una que incluya los recursos físicos, digitales, humanos y sociales que faciliten el acceso y uso digital significativos por parte de todos.

Palabras clave: brecha digital, literacidad digital, inclusión social, acceso, tecnología educativa

\begin{abstract}
While inequalities related to technology access and use are increasingly the focus of educational policy and practice, educators and policymakers alike continue to struggle to define and conceptualize the notion of a «digital divide». This divide could be conceived as a lack of access to technology and as a problem solved by simply providing Internet and computer access. As basic technology access has increased, attention has instead shifted to disparities in the broad array of factors that allow technologies to be effectively used. Rather than a technocentric framework that sees technology access alone as the solution to inequality, this paper draws from critical conceptualizations of print literacy in order to reframe the digital divide in terms of social inclusion through technology. The paper first provides examples of attempts to bridge the digital divide in India, Ireland, Egypt, and Peru. Based on these examples and other research, the paper then describes a framework for using technology for social inclusion. This framework aims to reorient the discussion of the digital divide toward one that includes the physical, digital, human, and social resources that facilitate meaningful digital access and use by all.
\end{abstract}

Keywords: digital divide, digital literacy, social inclusion, access, educational 


\section{Medios digitales e inclusión social}

La tecnología de la información y la capacidad de usarla y adaptarla es un «factor importante en la generación de riqueza, poder y conocimientos, así como en el acceso a estos en nuestros tiempos» (Castells, 1998, p. 92). Ello ha generado que tanto la sociedad como las escuelas centren gran atención en el acceso desigual a las nuevas tecnologías o la llamada brecha digital. Aunque la duda sobre $s i$ abordar las desigualdades relacionadas con el acceso y uso de la tecnología parece haber cambiado a una duda sobre cómo hacerlo, el problema sobre cómo conceptualizar y poner en marcha la brecha digital sigue presente. Un marco para la inclusión social a través de la tecnología, o inclusión electrónica, es una forma de abordar la brecha digital (Warschauer, 2002), pero ¿cómo funciona este marco dentro del contexto de políticas que enfatizan cada vez más la necesidad de la integración de la tecnología en la educación? El presente artículo, primero, describe cuatro ejemplos de intentos por superar la brecha digital en los contextos social y educativo. A partir de estos ejemplos y otras investigaciones, se discute un marco destinado a usar la tecnología en la inclusión social.

\section{Inclusión electrónica en cuatro contextos}

\section{1 «Educación mínimamente invasiva»}

El experimento «Agujero en la Pared» comenzó en el año 2000 como una manera de brindar acceso a computadoras y educación a niños de la calle en Nueva Delhi. El Gobierno de Nueva Delhi se asoció a una compañía especializada en tecnología de la información para instaurar quioscos con computadoras en barrios empobrecidos de Nueva Delhi. Estas estaciones contaban con cinco computadoras, las cuales se encontraban dentro de una cabina cerrada. Los monitores y un panel de palancas de mando y botones permitían a los niños visualizar e interactuar con las máquinas. No había teclados ni ratones. Asimismo, estos quioscos tenían acceso a Internet con conexión telefónica.

Basándonse en una idea llamada educación mínimamente invasiva, estos quioscos «Agujero en la Pared» solo ofrecían un apoyo mínimo. Un voluntario dentro del quiosco mantenía las computadoras y las conexiones de Internet; y, a la vez, era la extensión del soporte ofrecido. No había profesores o instructores presentes. En realidad, el objetivo de este experimento era permitir a los niños establecer su propio ritmo de aprendizaje, enseñar a sus compañeros y tener acceso las veinticuatro horas sin las restricciones de la supervisión y dirección de un adulto.

Los informes iniciales concluyeron que el proyecto había logrado atraer a los niños a los quioscos. Los niños aprendían solos a usar las funciones básicas de una computadora, cómo usar las palancas de mando y los botones para hacer clic y arrastrar objetos, navegar entre menús, usar programas como Microsoft 
Word, y copiar y pegar. También, pudieron acceder a Internet y cambiar el fondo del escritorio. El Gobierno afirmó que el proyecto había sido un éxito, y una investigación inicial sugirió que el programa era un éxito. Este último fue aclamado como un modelo de bajo costo potencialmente revolucionario para acercar el acceso a la tecnología y la educación a niños pobres.

Estas evaluaciones iniciales contrastaron con las visitas al quiosco inicial, las cuales tuvieron resultados diferentes. Si bien los niños dominaron las funciones simples como el uso de la palanca de mando y los botones, pasaban casi todo el tiempo usando algún software para dibujar y juegos para computadora. El acceso a Internet no era confiable y se usaba muy poco. No había disponible ningún software educativo. A ello se debe agregar que, aunque los niños solo conocían el hindi, no había ningún contenido especial en ese idioma. Asimismo, la distribución espacial del quiosco -diseñado como una pared en lugar de un espacio cerrado- obstaculizaba la colaboración y supervisión de los niños. Además, no hubo intentos por parte de los organizadores del programa para asociarse a organizaciones de la comunidad o educadores. En cuanto a los padres del vecindario, sus actitudes respecto a este quiosco eran variadas: algunos expresaban opiniones positivas, mientras que otros manifestaban su preocupación por la falta de supervisión e instrucción directa. Por ejemplo, un padre dijo que a su «hijo le iba muy bien en la escuela, se concentraba en sus tareas, pero que ahora pasaba todo su tiempo libre jugando en la computadora del quiosco y su rendimiento escolar había decaído».

A medida que el programa se expandía para incluir quioscos adicionales distribuidos en comunidades de pueblos más pequeños, estudios posteriores hallaron que este programa tenía limitaciones adicionales. Por ejemplo, las niñas visitaban los quioscos con menos frecuencia que los niños, y los niños rurales pasaban menos tiempo que sus pares urbanos en las computadoras (DeBoer, 2009). Otro estudio (Mitra y Dangwal, 2010) evaluó la presencia de un mediador local para asistir a los niños en un quiosco rural. Debido a que se buscaba mantener un esquema de proyecto mínimamente invasivo, el mediador no podía ser un profesor. Sin embargo, cabe anotar que este estudio encontró que la ayuda de un mediador tenía efectos positivos sobre el aprendizaje de los participantes. Estos hallazgos hacen eco de lo que se encontró en proyectos similares, como el programa Portales Digitales. Un estudio sobre este programa -cuyo objetivo era brindar acceso a computadoras a niños pobres de Sudáfrica- recomendó que los contextos de «aprendizaje no asistido» podrían beneficiarse con la presencia de facilitadores y educadores capacitados (Gush y de Villiers, 2010).

Si bien estos estudios tuvieron algunos efectos positivos, sigue habiendo serias dudas sobre la sostenibilidad de estos esfuerzos. Un estudio etnográfico de dos quioscos rurales «Agujero en la Pared» halló que ambos habían sido desmantelados (Arora, 2010). Ubicados en pueblos pequeños en 2002, estos quioscos tenían muy poco apoyo constante. Uno de ellos, en el pueblo 12 I de Almora, rápidamente cayó en desuso, debido al vandalismo; otro quiosco 
en Hawalbagh finalmente fue desconectado en 2007. Los «cuidadores» de los quioscos habían sido designados, pero "no se les pagó por mantener los equipos y no supieron de la organización [Agujero en la Pared] durante un mes» (Arora, 2010, p. 694). Los profesores, por su parte, manifestaron su desagrado por los quioscos, respecto a lo cual afirmaron: «está bien cuando las personas tienen conocimiento del uso de computadoras, pero cuando la mayoría de personas como aquí no lo tiene, necesitan orientación» (Arora, 2010, p. 693). Sin un apoyo técnico continuo y sin la participación de la comunidad, estos pueblos no se beneficiaban del «aprendizaje gratuito», que era la promesa del programa. Finalmente, los padres y la comunidad se dieron cuenta de que la «educación mínimamente invasiva» era, en la práctica, una educación mínimamente efectiva.

\subsection{Un pueblo en la Era de la Información}

Las organizaciones privadas pueden también iniciar esfuerzos para minimizar la brecha digital. En medio del crecimiento de su industria de producción de tecnología de la información y comunicación (TIC), Irlanda enfrentó desafíos relacionados con el uso limitado de las TIC entre sus ciudadanos y pequeñas empresas. En 1997, la empresa irlandesa de telecomunicaciones Telecom Eirann (llamada después Eircom) organizó un concurso para seleccionar, financiar y crear un «Pueblo en la Era de la Información» (Mooney, comunicación personal, 2001; McQuillan, 2002).

En este concurso, cualquier pueblo de Irlanda de 5000 personas o más podía presentar sus propuestas. Cada uno proponía su visión de lo que podría ser un pueblo en la era de la información y cómo podrían lograr esa transformación. El pueblo ganador se haría acreedor de 15 millones de libras irlandesas (22 millones de dólares americanos) para implementar su propuesta. Puesto que Telecom Eirann estaba en un proceso de privatización, la empresa tenía interés en escoger una propuesta con objetivos ambiciosos y tecnología avanzada de comunicaciones, debido a que, de esa manera, podría exhibir sus habilidades en la ejecución de un despliegue innovador y moderno de las telecomunicaciones. Se escogieron a 4 pueblos como finalistas; y, luego, Ennis -un pueblo pequeño y remoto de 15.000 personas, ubicado al oeste de Irlanda- fue elegido como el ganador. El premio monetario que Ennis recibió representaba más de 1200 dólares americanos por residente, una gran suma para un pueblo irlandés con dificultades.

Un aspecto clave de la propuesta ganadora de Ennis era un plan para ofrecer a cada familia del pueblo una computadora personal con acceso directo a Internet. Otras iniciativas incluían una página web para cada negocio que quisiera una, lectoras de tarjetas inteligentes para cada negocio (en aras de una sociedad sin dinero en efectivo) y tarjetas inteligentes para cada familia. Telecom Ireland alentó sobremanera a Ennis a implementar estos planes lo más pronto posible. Entretanto, los tres finalistas -los pueblos de Castlebar, 
Kilkenny y Kilarney- recibieron 1 premio consuelo de 1 millón de libras irlandesas (aproximadamente 1,5 millones de dólares americanos), y todo el tiempo que necesitaron para usar el dinero. Una visita a Ennis tres años más tarde por parte de un investigador universitario indicó que el pueblo tenía muy poco que mostrar si se consideraba el dinero recibido. La avanzada tecnología había sido entregada a personas con muy poca preparación. Se implementaron programas de capacitación, pero no fueron muy bien complementados por programas de concientización respecto a por qué las personas deben usar la nueva tecnología en primer lugar. En algunos casos, los sistemas sociales que funcionaban bien fueron perturbados para dar paso a la tecnología en exhibición. Por ejemplo, al igual que en el resto de Irlanda, los desempleados de Ennis se habían estado reportando a la oficina de beneficencia social tres veces por semana para inscribirse y recibir sus pagos. Luego de sus visitas, las personas normalmente permanecían en la oficina para conversar con otros trabajadores desempleados. En ese sentido, el sistema de inscripción facilitaba la importante función social de superar el aislamiento de los desempleados. Sin embargo, como parte del plan «un Pueblo en la Era de la Información», los residentes desempleados recibieron computadoras y conexiones de Internet en casa. Se les instruyó sobre cómo inscribirse y recibir pagos electrónicos vía Internet, en lugar de acercarse a la oficina a hacerlo. Pese a ello, muchos de los desempleados no lograban descifrar cómo hacer funcionar el equipo, y otros no veían sentido en hacerlo, pues los privaba de una importante oportunidad de socializar. Como consecuencia, se reportó que muchas de esas computadoras se vendieron en el mercado negro y los desempleados simplemente volvían por iniciativa propia a la oficina de la beneficencia social para inscribirse.

En cuanto a los otros tres pueblos que recibieron muchísimos menos recursos, se vieron obligados a planificar con mucho cuidado el uso que le darían a esos fondos, en lugar de malgastar cantidades abismales en equipos caros. Los socios, conformados por grupos comunitarios, empresas pequeñas y sindicatos de trabajadores, fueron parte del proceso. Se invirtió mayor esfuerzo y dinero en concientizar, planificar e implementar una capacitación efectiva y establecer procesos para un cambio sostenible en lugar de una simple compra de equipos. Los pueblos se apoyaron en redes existentes de trabajadores, educadores y empresarios para respaldar los usos básicos de la tecnología en el desarrollo social y económico. La información sobre servicios sociales y oportunidades de empleo estuvo disponible en línea. Las empresas pequeñas y los artesanos, por su parte, aprendieron a agrupar sus recursos para promover sus productos a través del comercio electrónico. Asimismo, se designaron coordinadores de tecnología en las escuelas, que trabajaban con otros profesores para desarrollar planes para una mejor integración de la TIC en las aulas. Finalmente, según un investigador de University College Dublin (Mooney, comunicación personal, 2001), los tres finalistas que recibieron solo $1 / 15$ del dinero recibido por Ennis tenían más que mostrar por sus esfuerzos para promover la inclusión social a través de la tecnología que el propio ganador. 
Desafortunadamente, aún existen muchos vacíos en el acceso y el uso de Internet. Cerca de un tercio de adultos irlandeses acceden a Internet una vez a la semana o menos, mientras que uno de cada cinco nunca lo ha usado (Ireland Department of Communications, Energy, and Natural Resources [DCENR] ${ }^{1}$, 2013). A pesar del creciente comercio electrónico en Irlanda, solo un 22,9\% de las pequeñas empresas participaban en transacciones en línea en el 2012 (DCENR, 2013, p. 6).

A partir del experimento «un Pueblo en la Era de la Información», los esfuerzos por incentivar el uso de la tecnología aumentaron. En 2013, el Ministro de Comunicaciones de Irlanda, Pat Rabbitte, lanzó la Estrategia Digital Nacional (DCENR, 2013). En julio de 2013, se anunció una iniciativa de 1,4 millones de euros que, inicialmente, tenía el fin de capacitar a la comunidad en el uso de Internet. Esta iniciativa tiene como objetivo ayudar a las pequeñas empresas a usar transacciones en línea, reducir el número de personas que aún no se han conectado a Internet y desarrollar infraestructura de TIC, desarrollo profesional y apoyo curricular a las escuelas. Estos esfuerzos son parte de un esfuerzo holístico para mejorar la inclusión de los ciudadanos, no solo en términos de acceso y uso de Internet, sino también en términos de asistencia médica, participación de la comunidad y crecimiento económico. Básicamente, el objetivo de la iniciativa es crear una nación en la era de la información.

\subsection{Un laboratorio modelo de computadoras}

Un proyecto de un donante internacional, financiado por la Agencia de los Estados Unidos para el Desarrollo Internacional (Usaid), decidió donar un laboratorio de computadoras a la escuela de educación de una gran universidad egipcia (para mayores detalles de este programa, véase Warschauer, 2004). El propósito de la donación fue establecer un programa modelo para capacitar a maestros en el aprendizaje asistido por computadoras en una de las áreas de la escuela. Se escogieron equipos modernos, incluyendo más de cuarenta computadoras, un carísimo sistema de proyección de videos, varias impresoras y escáneres, y decenas de miles de dólares americanos en software educativo. Este debía ser un proyecto modelo que los gobiernos de los Estados Unidos y Egipto mirarían con orgullo. Para garantizar la sostenibilidad del mismo, era necesario que la universidad egipcia manejara todos los gastos y operaciones continuos, incluyendo el pago del acceso a Internet, el mantenimiento de la red de área local (LAN) y el funcionamiento del laboratorio de computadoras.

Siguiendo un contrato pagado por Usaid, un comité de la escuela de educación dentro de la universidad egipcia preparó una propuesta detallada sobre cómo se usaría, funcionaría y mantendría el laboratorio. Sobre esta base, Usaid compró todo el hardware y software. Sin embargo, antes de instalar los equipos,

1 Se utiliza estas siglas por su nombre en castellano: Departamento de Comunicaciones, Energía y Recursos Naturales de Irlanda. 
fue más que evidente que la escuela tendría dificultades en absorber una donación tan grande y cara. Otros departamentos al interior de la escuela -que, en conjunto, tenían acceso a solo unas pocas computadoras- comenzaron a envidiar que un solo departamento tuviese equipos tan modernos y caros, e intentaron bloquear el apoyo de la universidad al laboratorio. Ni la escuela ni la universidad pudieron justificar con facilidad el gasto del dinero para albergar y mantener un laboratorio tan caro en un solo programa cuando los demás programas tenían poco financiamiento. Además, no había dinero disponible para contratar a un administrador del LAN u ofrecer acceso a Internet al nivel acordado en la propuesta. Asimismo, hubo problemas en las relaciones de los profesores de la universidad, pues el presidente de un departamento clave tomó a mal la participación e iniciativa de profesores de menor rango que formaron parte de la capacitación y trabajaban juntos en la planificación del nuevo currículo. Debido a todas estas dificultades, las caras y modernas computadoras se quedaron en sus cajas en una oficina con llave por más de un año sin ser instaladas, por lo cual -además- perdieron un tercio de su valor económico.

Cuando finalmente se implementó el laboratorio, este se convirtió en una «notable excepción» respecto a los otros laboratorios de computadoras de Egipto; sin embargo, su éxito fue pasajero (England, 2007, p. 383). Mientras que muchos laboratorios carecían de profesores y acceso confiable a Internet, este laboratorio de computadoras tenía ambos. A partir de la comunicación con los profesores del laboratorio, se planteó que «hasta hace muy poco cuando el laboratorio "se venía abajo", según un profesor, el laboratorio era un modelo de instrucción» (England, 2007, p. 384). No obstante, las dificultades técnicas y la posterior pérdida de acceso a Internet en el laboratorio significaron que el espacio ya no podía soportar la instrucción. Al igual que muchos proyectos de ayuda bien intencionados, demostró ser insostenible en el contexto local.

\subsection{Una educación moderna}

En 2007, el Perú se asoció a la Corporación Una Laptop Por Niño (ULPN en castellano, u OLPC, por sus siglas en inglés) para ofrecer laptops individuales a estudiantes del programa. Esta iniciativa fue propuesta y diseñada por Óscar Becerra, Director General de Tecnología de la Educación del Ministerio de Educación del Perú, cuyo objetivo era «modernizar» la educación y permitir a «los niños y profesores acceder a la TIC y explorarla de una manera no amenazante» (Becerra, 2010). De este modo, se adquirieron las primeras 290.000 laptops para ser distribuidas entre los niños. Finalmente, se distribuyeron más de 900.000 laptops entre niños y escuelas, lo cual constituyó a ULPN como un compromiso enorme y caro.

Frente a ello, la pregunta es la siguiente: ¿Esta iniciativa, de entregar una laptop a los estudiantes, ha brindado la educación «moderna» que se esperaba? Un estudio independiente (Derndorfer, 2010) y una evaluación dirigida 16 I por el Banco Interamericano de Desarrollo (Santiago, Severin, Cristia, Ibarra- 
rán, Thompson y Cueto, 2010) encontraron que esta iniciativa rápidamente se topó con dificultades de infraestructura. Múltiples escuelas rurales carecían de electricidad, mientras que otras escuelas que sí contaban con electricidad a veces solo tenían un tomacorriente en la oficina del director, lo que implicaba que la carga -y el posterior uso- de las laptops fuera casi imposible. La falta de acceso a Internet en la mayoría de las escuelas limitó aún más la forma como las laptops podían ser usadas. La falta de soporte técnico y desarrollo profesional de los profesores también era un problema. Solo un 10,5\% de los profesores reportaron haber recibido soporte técnico, y solo un 7\% confirmó haber recibido soporte pedagógico para usar las laptops en las aulas. Los docentes de escuelas rurales de una sola aula casi nunca podían viajar para recibir capacitación, debido a sus responsabilidades en la escuela o falta de voluntad para recibir capacitación no pagada durante sus vacaciones. En ese sentido, las laptops no incentivaron el logro académico en los lugares en los que fueron instaladas (Beuermann, Cristia, Cruz-Aguayo, Cueto y Malamud, 2013; Cristia, Ibarrarán, Cueto, Santiago, y Severín, 2012). Asimismo, se debe agregar que un estudio halló que ULPN inicialmente hizo muy pocos esfuerzos por satisfacer las necesidades de la diversa población del Perú, especialmente, respecto a la valoración del conocimiento local y la literacidad de los pueblos indígenas (Breitkopf, 2012).

Para tratar estos problemas, el programa ha cambiado de rumbo desde entonces. ULPN priorizó a las escuelas que contaban con electricidad e infraestructura de Internet (Cristia et ál., 2012). A inicios del 2010, las XO se distribuyeron entre las escuelas en lugar de estudiantes individuales, con el fin de extender la distribución a más escuelas (Breitkopf, 2012, p. 68). En ese contexto, mientras expresaba su decepción por la falta de planificación del programa ULPN, el Director General de Tecnología Educativa de ese momento, Sandro Marcone, anunció que el Ministerio de Educación del Perú ya no realizaría más compras de computadoras a gran escala (Derndorfer, 2012). Pese a un costo muy elevado para el Gobierno, ULPN no cumplió con su objetivo de implementar una educación «moderna».

\section{Una brecha digital reconceptualizada}

Cada uno de los programas antes descritos estuvo motivado por un sincero intento de mejorar las vidas de las personas a través de las TIC. No obstante, cada programa se topó con dificultades inesperadas, que obstaculizaron los resultados. La iniciativa de las laptops en el Perú se enfrentó a problemas de infraestructura, una falta de conexión entre los objetivos del programa y las necesidades de su población. De manera similar, el experimento «Un Pueblo en la Era de la Información» valoró el despliegue de la nueva tecnología por encima de las necesidades de la comunidad. En el caso del experimento «Agujero en la Pared», se enfatizó en el despliegue de la tecnología ante la exclusión completa de la pedagogía. 
Evidentemente, todo proyecto de TIC es complicado, y no se debe esperar que ninguno funcione fluidamente. Sin embargo, los problemas con estos proyectos no fueron ni aislados ni azarosos. Más bien, estos tipos de problemas ocurren una y otra vez en proyectos de tecnología en todo el mundo: a menudo, se centran en la provisión de hardware y software, pero prestan poca atención a los sistemas humanos y sociales que también deben cambiar para que la tecnología pueda hacer alguna diferencia. Tal como se aprecia en estas tres historias, el importante acceso a las TIC comprende más que una simple entrega de computadoras y conexiones de Internet. En realidad, el acceso a las TIC está inscrito en un complejo conjunto de factores, comprendido por recursos y relaciones físicas, digitales, humanas y sociales. En ese contexto, se deben tomar en cuenta el contenido y el idioma, la literacidad y la educación, y las estructuras comunitarias e institucionales si se va a ofrecer un importante acceso a las nuevas tecnologías.

¿Puede la brecha digital capturar estos factores completamente? Quizás, la brecha digital podría ser una brecha extendida, a fin de comprender este amplio conjunto de factores y recursos. En ese sentido, una brecha digital se caracteriza no solo por el acceso físico a computadoras y conectividad, sino también por el acceso a recursos adicionales que permitan a las personas usar la tecnología correctamente. No obstante, el sentido original del término brecha digital-que concedía una importancia predominante a la disponibilidad física de las computadoras y la conectividad, en lugar de asuntos que tienen que ver con recursos de contenido, idioma, educación, literacidad o recursos comunitarios y sociales- es difícil de superar en las mentes de las personas.

Un segundo problema con el concepto de brecha digital es su implicancia en la división social binaria. Como manifiesta Cisler (2000), no existe una división binaria entre «los que poseen» $\mathrm{y}$ «no poseen» información, sino más bien un espectro basado en diferentes grados de acceso a la tecnología de la información. Compárese, por ejemplo, un profesor de UCLA con conexión de alta velocidad en su oficina, un alumno en Seúl que de vez en cuando acude a un cibercafé y una activista rural de Indonesia que no cuenta con una computadora o línea telefónica, pero cuyas colegas en su grupo de mujeres descargan e imprimen información para ella. Este ejemplo ilustra solo tres grados del posible acceso que una persona puede tener a material en línea. Respecto a ello, Hargittai y Walejko (2008) sugieren que la desigualdad que proviene del uso de la tecnología es en realidad una brecha de la participación. Este «segundo nivel de brecha digital» se caracteriza por habilidades, usos y experiencias diferenciadas con la tecnología (Hargittai, 2002). En las escuelas de los Estados Unidos, estas diferencias de habilidades son particularmente evidentes entre alumnos de un estatus socioeconómico bajo y de una minoría subrrepresentada (Warschauer y Matuchniak, 2010).

La noción de una brecha binaria entre los que tienen y los que no es, entonces, inexacta, e incluso puede ser condescendiente por cuanto no valora los 
los Estados Unidos, la brecha digital a menudo se representa en términos de diferencias entre grupos raciales. Sin embargo, la heterogeneidad dentro de los grupos raciales es, por lo general, mayor que entre los grupos sociales. Solo un $49 \%$ de familias negras urbanas de pocos ingresos (que ganan menos de US\$ 25.000) usan computadoras en comparación con un $90 \%$ de las familias urbanas que ganan US $\$ 75.000$ o más (National Telecommunications and Information Administration ${ }^{2}$, 2011). Vacíos similares existen respecto a la adopción de una banda ancha, en la que los índices de adopción de las familias urbanas blancas van de $49 \%$ a $92 \%$, dependiendo del grupo de ingresos.

La noción de una brecha digital -incluso en su sentido más amplio- implica una cadena de causalidad. Como metáfora, la brecha digital implica que la falta de acceso (independientemente de su definición) a computadoras e Internet perjudica las oportunidades en la vida de un individuo. Si bien esto último es indudablemente cierto, lo opuesto es igual de cierto: aquellos que ya están marginalizados tendrán menos oportunidades para acceder y usar computadoras e Internet. De hecho, la tecnología y la sociedad están entrelazadas y se complementan, y esta compleja relación hace que cualquier presunción de causalidad sea problemática.

Finalmente, se debe considerar que el marco de la brecha digital ofrece una hoja de ruta deficiente en el uso de la tecnología para promover el desarrollo social, puesto que enfatiza de manera excesiva la importancia de la presencia física de las computadoras y la conectividad, y excluye otros factores que permiten a las personas a usar las TIC para fines significativos. Rob Kling, director del Centro de Informática Social de la Universidad de Indiana, explica muy bien esta falla:

[El] gran problema del marco de "la brecha digital" tiene que ver con que tiende a connotar "soluciones digitales", es decir, computadoras y telecomunicaciones, sin involucrar el importante conjunto de recursos complementarios e intervenciones complejos que apoyan la inclusión social, de los cuales las aplicaciones de la tecnología de la información pueden ser elementos permisivos, pero son ciertamente insuficientes cuando simplemente se suman al status quo de la mezcla de recursos y relaciones (Warschauer, 2003b, p. 7-8).

En conclusión, no existe una brecha binaria, así como ningún factor predominante que determine dicha brecha.

De esta manera, se resalta que la brecha digital es una metáfora inadecuada para abordar el tema de la desigualdad. La TIC no existe como una variable externa que se inyecta desde el exterior para ocasionar ciertos resultados. Más bien, está interconectada de manera compleja con los sistemas y procesos sociales. Desde una perspectiva de políticas, el objetivo de usar las TIC en grupos 
marginalizados no es superar una brecha digital, sino más bien promover un proceso de inclusión social. Para lograrlo, es necesario «centrarse en la transformación, no en la tecnología» (Jarboe, 2001, p. 31). A la vez que se reconoce el valor histórico del concepto de la brecha digital como un medio para centrar la atención en un importante tema social, el presente artículo propone en realidad conceptos y terminología alternativos, que describen de una forma más precisa los temas que están en juego y los retos sociales futuros.

\section{Un marco para la inclusión digital}

Este marco alternativo propuesto es el de la tecnología para la inclusión social. La inclusión y la exclusión social se refieren al ámbito en el cual los individuos, las familias y las comunidades pueden participar a cabalidad en la sociedad y controlar sus propios destinos, para lo cual se considera una variedad de factores relacionados a recursos económicos, empleo, salud, educación, vivienda, recreación, cultura y participación social. En el contexto de la tecnología y la educación, estas han sido discutidas en términos de la «inclusión digital» (Selwyn, 2004; Livingstone y Helsper, 2007; Salinas y Sánchez, 2009; Warschauer, 2002).

La inclusión social no solo implica compartir adecuadamente los recursos, sino también "participar en la determinación de las oportunidades en la vida individual y colectiva» (Stewart, 2000). Ello se superpone con el concepto de igualdad socioeconómica, mas no es su equivalente. Existen muchas maneras a partir de las cuales las personas en situación de pobreza pueden tener una mayor participación e inclusión, incluso si no tuvieran igualdad de recursos. Al mismo tiempo, se debe considerar que aun aquellos a los que les va muy bien pueden enfrentar problemas de exclusión social, debido a razones de persecución política o discriminación por cuestiones de edad, género, preferencia sexual o discapacidad. El concepto de inclusión social no ignora el rol de la clase, sino que reconoce que el amplio espectro de otras variables ayuda a determinar la interacción de las fuerzas de clases. Si bien el tratamiento histórico del término va más allá del ámbito del presente artículo, uno podría sostener que el concepto de inclusión social refleja particularmente bien los imperativos de la era actual de la información, donde temas como identidad, idioma, participación social, comunidad y sociedad civil son el centro del debate (Castells, 1997).

\section{Modelos de acceso e inclusión}

\subsection{Modelos tecnocéntricos}

¿Qué papel, entonces, puede jugar la tecnología en la promoción de la inclusión social? Eso depende en gran medida de nuestra definición de «acceso». El modelo más común para pensar en el acceso a la tecnología es el que se basa en la propiedad o disponibilidad de un aparato, en este caso, una computado- 
ra. Los aparatos físicos pueden difundirse relativamente rápido $\mathrm{y}$, en algunos casos, de manera equitativa; nótese, por ejemplo, el casi grado universal de propiedad de televisores en los Estados Unidos, tanto entre pobres como ricos. Sin embargo, el modelo del aparato tiene varias fallas, comenzando por el hecho de que el precio real de la compra de una computadora es solo una pequeña parte de lo que puede considerarse como el costo total de la propiedad. Esto último incluye el precio del software, el mantenimiento, las periferias y, en contextos institucionales, capacitación, planificación y administración (véase a Patterson y Wilson III, 2000), sin mencionar el costo de reemplazar el hardware y software debido a la obsolescencia del producto según la planificación de la corporación.

No obstante, más importante aún es que otras barreras que van más allá de la asequibilidad de computadoras (o de paquetes de computación más extensos) seguirán jugando un papel importante en el fomento de la desigualdad digital. Estas barreras incluyen el acceso diferenciado a telecomunicaciones de banda ancha; diferencias en el conocimiento y las habilidades al usar las computadoras, o en las actitudes respecto a su uso; el inadecuado contenido que está disponible en línea para las necesidades de ciudadanos de bajos ingresos, especialmente, en diversos idiomas; y los controles o las limitaciones gubernamentales sobre el uso irrestricto del Internet en muchas partes del mundo (véase la discusión en DiMaggio, Hargittai, Celeste y Shafer, 2004).

Debido a estas limitaciones, el modelo del aparato ha sido mejorado de alguna manera gracias al modelo del conducto (véase Lievrouw, 2000). Si bien se puede adquirir un aparato en una sola compra, el acceso al conducto necesita estar conectado a una línea de abastecimiento que ofrezca algo de forma regular, como electricidad, servicio telefónico o televisión por cable. La difusión de los conductos es más lenta que la de los equipos, ya sea porque primero se debe establecer una infraestructura de distribución (como la instalación de líneas telefónicas o cables de fibra óptica) o porque el costo de una tarifa mensual es un elemento disuasorio para el acceso. Por ejemplo, los conductos como electricidad, servicio telefónico y servicio de televisión por cable se han difundido más lentamente que los aparatos como televisores, radios y filmadoras.

Asimismo, se debe anotar que la difusión de conductos a menudo involucra un alto grado de movilización y lucha social para asegurar el acceso equitativo. Esto ocurrió de manera más notable con la electricidad, pues muchos países escogieron diferentes caminos para masificar (o hacer más selectiva) la electrificación, en gran parte, debido al equilibrio de las fuerzas sociales y de clase en el país (véase la discusión de Brown, 1980). De manera similar, en muchas naciones, las largas luchas sociales han ocurrido en nombre del acceso universal a los teléfonos.

Sin embargo, aunque los conductos representan un mejor modelo comparativo que los aparatos en el caso de las TIC, ninguna categoría captura la esencia del acceso significativo a las tecnologías de la información y comunicación. Lo que resulta más importante sobre las TIC no es tanto la disponibilidad de 
los aparatos de computación o la línea de Internet, sino más bien la capacidad de las personas para usar ese aparato o línea y, de esa manera, participar en prácticas sociales significativas. Las personas que no pueden leer, que nunca aprendieron a usar una computadora y que no conocen ninguno de los más importantes idiomas que dominan el software disponible y el contenido en Internet tendrán dificultad incluso para conectarse o usar el Internet de manera productiva.

\subsection{Modelos de literacidad}

El concepto de literacidad representa un mejor modelo de acceso. Mientras que la definición de literacidad según el sentido común es la habilidad individual de poder leer y escribir, muchos teóricos prefieren una definición más amplia que toma en cuenta los contextos sociales de la práctica de la literacidad. Señalan que lo que se considera una lectura o escritura habilidosa varía tremendamente según el contexto histórico, político y sociocultural (Gee, 2007). Se puede observar, por ejemplo, el conocidísimo caso de cambiar las prácticas letradas antes y después de la difusión de la prensa escrita (véase la discusión de McLuhan, 2011; Eisenstein, 1980), o las diferencias entre los tipos de literacidad apreciados en una madrassa pakistaní (escuela religiosa) en comparación con una universidad en los Estados Unidos. En este sentido más amplio, entonces, la literacidad implica «tener dominio sobre los procesos por medio de los cuales se codifica la información culturalmente significativa» (De Castell y Luke, 1986, p. 374).

Respecto a ello, se debe resaltar que existen muchas similitudes entre la literacidad y el acceso a las TIC (véase la tabla 1). En primer lugar, tanto la literacidad como el acceso a TIC están muy relacionados a los avances de la comunicación humana y los medios de producción de conocimientos. En segundo lugar, así como el acceso a TIC es un prerrequisito para la participación total en el campo informático del capitalismo, la literacidad fue (y sigue siendo) un prerrequisito para la participación cabal en los momentos iniciales industriales del capitalismo. En tercer lugar, la literacidad y el acceso a TIC necesitan una conexión a un artefacto físico (es decir, un libro o una computadora), a fuentes de información que se expresan como contenido dentro o a través de ese artefacto físico, $y$ a un nivel de habilidades suficiente para procesar y hacer uso de esa información. En cuarto lugar, ambos implican no solo la recepción de información, sino también su producción. Finalmente, los dos están relacionados a nociones algo controversiales en torno a las brechas sociales: la gran brecha de la literacidad y la brecha digital. 
Tabla I. Comparación de la literacidad y el acceso a las TIC

\begin{tabular}{|c|c|c|}
\hline & Literacidad & Acceso a las TIC \\
\hline $\begin{array}{l}\text { Etapa de } \\
\text { comunicación }\end{array}$ & Escritura y texto impreso & $\begin{array}{l}\text { Comunicación mediante } \\
\text { computadoras }\end{array}$ \\
\hline Era económica & Capitalismo industrial & Capitalismo informacional \\
\hline Artefacto físico & $\begin{array}{l}\text { Libros, revistas, periódicos, } \\
\text { revistas especializadas }\end{array}$ & $\begin{array}{l}\text { Aparatos (computadoras, } \\
\text { tablets, smartphones) }\end{array}$ \\
\hline $\begin{array}{l}\text { Organización del } \\
\text { contenido }\end{array}$ & $\begin{array}{l}\text { Libros literarios, historietas, } \\
\text { ensayos, informes, poemas y } \\
\text { formularios. }\end{array}$ & $\begin{array}{l}\text { Páginas web, correo electró- } \\
\text { nico, mensajes instantáneos, } \\
\text { medios sociales }\end{array}$ \\
\hline $\begin{array}{l}\text { Habilidades } \\
\text { receptivas }\end{array}$ & Lectura & $\begin{array}{l}\text { Lectura }+ \text { interpretación de } \\
\text { multimedia, buscar y navegar } \\
\text { en Internet }\end{array}$ \\
\hline $\begin{array}{l}\text { Habilidades } \\
\text { productivas }\end{array}$ & Escritura & $\begin{array}{l}\text { Escritura + autoría y } \\
\text { publicación de multimedia }\end{array}$ \\
\hline Brechas & $\begin{array}{l}\text { ¿Una gran brecha de } \\
\text { literacidad? }\end{array}$ & ¿Una brecha digital? \\
\hline
\end{tabular}

Fuente: Elaboración propia

\section{La brecha de la literacidad}

Al igual que las habilidades y el acceso digitales, la literacidad impresa comprende una gama de habilidades, conocimientos y acceso a medios físicos y a los canales que sirven para producir esos medios. La literacidad se distribuye y practica sobre una base altamente desigual, y se correlaciona sobremanera con los ingresos y la riqueza a nivel individual y social. También, por ello, la importancia de la literacidad en el desarrollo social e individual es ampliamente reconocida. Estas características hacen eco a las de la literacidad digital, marco en el que el acceso y uso desiguales se asocian a pobres logros académicos (Warschauer y Matuchniak, 2010). ¿Qué ganamos al ver la brecha digital desde el lente de la brecha de la literacidad?

Al discutir la gran brecha de la literacidad, surge el tema de la causalidad: ¿la literacidad permite el desarrollo, o el desarrollo desigual (y su correspondiente distribución desigual del poder político, económico y social) impide el acceso de las personas a la literacidad? Algunos defensores de la primera noción proponen la existencia de una brecha de la literacidad. Desde esta perspectiva, no existen diferencias cognitivas fundamentales en los individuos alfabetizados y los que no lo son, que den como resultado una gran brecha en la literacidad en los niveles individual y social. Se dice que la literacidad ha separado a la prehistoria de la historia (Goody y Watt, 1963), a las sociedades primitivas de las civilizadas (Levi Strauss, en Charbonnier, 1973), y a las so- 
ciedades modernas de las tradicionales (véase la discusión de Scribner y Cole, 1981). A nivel individual, se dice que la literacidad permite a las personas dominar las funciones lógicas del idioma (Goody, 1975; Olson, 1977) y pensar de manera abstracta (Greenfield, 1972; Luria, 1976). Puesto que la literacidad, los ingresos familiares y la educación se correlacionan sobremanera, es difícil estudiar los beneficios cognitivos de ser alfabetizado.

Respecto a ello, dos psicólogos de la educación, Sylvia Scribner y Michael Cole, elaboraron una solución creativa al problema de investigación para determinar los beneficios cognitivos particulares de la literacidad en el aislamiento de sus covariables. Encontraron una tribu en Liberia, los Vai, que desarrolló su propia transcripción escrita en el idioma local de la tribu. La literacidad de los escritos Vai se transmitía a través de tutorías informales, mas no por una enseñanza formal. Asimismo, se debe anotar que la escritura Vai era utilizada de manera muy limitada, principalmente, para correspondencia personal y registros comerciales. A partir de ello, plantearon un estudio de tres grupos que comparaba a los miembros analfabetos de la tribu, a los alfabetos solo en el idioma Vai (a través de tutoría personal) y aquellos con mayores habilidades de literacidad en inglés o árabe obtenidas por medio de la enseñanza. Mediante esta división, Scribner y Cole (1981) pudieron separar los beneficios cognitivos que podrían probablemente ser atribuidos a la literacidad y los que fueron probablemente atribuidos a un ambiente más amplio de educación formal.

Como resultado, Scribner y Cole (1981) prácticamente no encontraron beneficios cognitivos generalizables en la literacidad Vai. Las diferencias individuales en una gama de tareas cognitivas, en áreas de abstracción, clasificación, memoria y lógica, se debieron más bien a otros factores, como la enseñanza o, en algunos casos, a vivir en un área urbana (en comparación con la rural). De este modo, el estudio de Scribner y Cole (1981) ayudó a formular la pregunta acerca de la existencia de una gran brecha de literacidad, al menos a nivel individual. Su trabajo mostró que no existe literacidad alguna que divida a las personas en dos campos cognitivos, sino que más bien existen gradaciones y tipos de literacidad con una gama de beneficios muy estrechamente relacionados a las funciones específicas de las prácticas de literacidad. Puede decirse que la literacidad no ocasiona el desarrollo social o cognitivo, sino que la literacidad y el desarrollo social están vinculados y reconstituidos, como lo están las tecnologías y la sociedad en general. En esa medida, la literacidad no es simplemente una cuestión de acceso, sino de inclusión en un entorno de prácticas sociales.

Si la literacidad debe ser reconceptualizada como práctica inmersa dentro de contextos sociales, entonces, la brecha digital podría beneficiarse de un replanteamiento similar en términos de igualdad, acceso y adquisición de habilidades. 


\section{La adquisición de la literacidad}

Si la literacidad se comprende como un grupo de prácticas sociales en lugar de una habilidad cognitiva restringida, esta tiene varias consecuencias importantes referentes a la adquisición de la literacidad, y paralelismos importantes con la adquisición del acceso a las TIC. La adquisición de la literacidad, como el acceso a TIC, requiere de una variedad de recursos. Estos incluyen artefactos físicos (libros, revistas, periódicos, revistas especializadas, computadoras, etc.); contenidos relevantes transmitidos por medio de esos artefactos; habilidades apropiadas del usuario, conocimiento y actitud; y los tipos adecuados de apoyo social y de la comunidad.

La disponibilidad física de los libros u otros materiales de lectura es sin duda esencial para la adquisición de la literacidad; sin embargo, los otros recursos son también importantes. Respecto a un contenido accesible y relevante, uno de los mayores obstáculos para adquirir la literacidad es la escasez de material publicado en muchos -sino en la mayoría- de los 7000 idiomas que se hablan en el mundo. Además, Paulo Freire (2000) y otros han demostrado que la instrucción de literacidad es más efectiva cuando se trata de un contenido referido a las necesidades y condiciones sociales de los que aprenden. En cuanto al material relacionado a las TIC, este contenido por lo general lo desarrollan mejor los propios aprendices.

La adquisición de la literacidad requiere, sin duda, el desarrollo de una variedad de habilidades, conocimientos y actitudes, incluyendo las habilidades del proceso cognitivo; el conocimiento general del mundo; y la motivación, el deseo y la seguridad para leer. Esto tiene paralelismos importantes con los tipos de habilidades, conocimientos y actitudes necesarios para el uso significativo de las TIC.

Finalmente, aprender a leer es un acto social que se cruza en una infinidad de formas con estructura social, organización social y prácticas sociales. Las personas aprenden a leer (y a leer de cierta manera) cuando se encuentran rodeados de otras que les brindan su apoyo en el proceso; desde los padres que les leen, compañeros de clase con los que comentan las revistas de historietas, hasta los ancianos de la aldea que valoran la educación de los niños. La naturaleza multifacética de la literacidad, la gama de recursos que requiere y la naturaleza social de su práctica y dominio hacen concluir que la adquisición de la literacidad no es solo una cuestión cognitiva, o incluso cultural, sino también de poder y política (Freire, 2000; Freire y Macedo, 2013; Gee, 2007; Street, 1995). En países como Sudáfrica, Brasil y hasta en los guetos empobrecidos de los Estados Unidos, el acceso a la literacidad se encuentra con oportunidades desiguales, referentes a la asistencia a la institución educativa, la distribución inequitativa de recursos dentro del sistema educativo, y del currículo y la pedagogía que satisfacen las necesidades de ciertos grupos sociales más que de otros. Tal vez, la evidencia más obvia de este fenómeno es el lamentable bajo porcentaje de literacidad de mujeres en muchos países del mundo en la ac- 
tualidad. Debido a la naturaleza politizada de la literacidad, las campañas que se centran exclusivamente en la habilidad individual e ignoran los sistemas sociales más amplios que apoyan o restringen la extensión de la literacidad no siempre son las más efectivas. En muchos casos, la literacidad en general no proviene de arriba, sino que es aprovechada desde abajo a través de la movilización social y la acción colectiva de los pobres y desposeídos.

\section{Acceso y literacidad}

Una síntesis de la discusión anterior brinda seis conclusiones importantes acerca de la literacidad, que pueden ser utilizadas como bases para un modelo de acceso a las TIC: a) No solo existe uno sino muchos tipos de literacidad; b) El significado y los valores de la literacidad varían en contextos sociales particulares; c) Las capacidades para la literacidad existen en gradaciones antes que en oposiciones bipolares; d) La literacidad por sí sola no brinda un beneficio automático fuera de sus funciones particulares; e) La literacidad es una práctica social que involucra el acceso a los artefactos físicos, el contenido, las habilidades y el apoyo social; y e) La adquisición de la literacidad no es solo cuestión de educación, sino también de poder.

Igualmente, se debe resaltar que no existe un solo tipo de acceso a las TIC sino muchos. El significado y el valor del acceso varían en contextos sociales particulares; el acceso existe en gradaciones antes que en una oposición binaria. Asimismo, se debe considerar que el uso de computadoras e Internet no brindan un beneficio automático fuera de las funciones particulares. Ello responde a que el uso de las TIC es una práctica social, que se conecta con el acceso a artefactos físicos, contenido, habilidades y apoyo social. Finalmente, cabe anotar que la adquisición del acceso a las TIC no es solo cuestión de educación, sino también de poder.

En ese contexto, el acceso a las TIC para la promoción de la inclusión social no puede quedarse en la provisión de aparatos o conductos solamente. Más bien, requiere del compromiso de una gama de recursos, todos desarrollados y promovidos con miras a mejorar el poder social, económico y político de los clientes y comunidades implicados. Cualquier intento por categorizar estos recursos es por naturaleza arbitrario, pero un análisis basado en las cuatro categorías generales sirve tanto para el análisis como para la formulación de políticas. Estas categorías han surgido de la investigación etnográfica (Warschauer, 1999, 2003a) y del estudio de casos (Grimes y Warschauer, 2008; Warschauer y Ames, 2010), y han sido señaladas en términos similares por otros teóricos interesados en el rol de la tecnología en la inclusión social (véase, por ejemplo, Aichholzer y Schmutzer, 2001; Carvin, 2000). A continuación, se presentan y detallan dicha categorías: a) Recursos físicos, b) Recursos digitales, c) Recursos humanos y d) Recursos sociales.

Los recursos físicos abarcan el acceso a computadoras y conexiones de 26 I telecomunicación. En cada una de las cuatro viñetas, los esfuerzos iniciales por 
incrementar el acceso a las TIC se centraron principalmente en los recursos físicos. Los recursos digitales, por su parte, se refieren al material digital que se encuentra disponible en línea. El acceso a las TIC es poco significativo si no permite que los usuarios encuentren el contenido digital relevante en términos de su contextualización. Con respecto a los recursos humanos, estos giran alrededor de temas tales como la literacidad y la educación (incluyendo los tipos particulares de prácticas letradas que son necesarios para el uso de la computadora y la comunicación en línea). En el Perú, por ejemplo, se brindó desarrollo profesional a profesores para que puedan usar mejor las computadoras en las aulas. Este esfuerzo se frustró en parte por la escasez de recursos sociales. Estos últimos hacen referencia a la comunidad, las estructuras sociales e institucionales que apoyan al acceso a las TIC. Los temas estructurales que producen un alto movimiento de profesores y la incapacidad de los mismos para poder tomarse el tiempo para desarrollos profesionales influyó sobre la insuficiencia de las capacitaciones.

Al considerar estos cuatro tipos de recursos, es importante darse cuenta de su relación iterativa con el uso de las TIC. Por un lado, cada uno de estos recursos es un contribuyente al uso efectivo de las TIC. En otras palabras, la presencia de estos recursos ayuda a asegurar que las TIC puedan ser bien utilizadas y aprovechadas. Por otro lado, el acceso a cada uno de estos recursos es un resultado del uso efectivo de las TIC. Esto significa que, al dar un buen uso a las TIC, se puede ayudar a extender y promover el acceso a estos recursos. $\mathrm{Si}$ el uso es bueno, estos recursos pueden entonces servir como un círculo virtual que promueve la inclusión y el desarrollo social. Si el uso es deficiente, estos elementos pueden servir como ciclo vicioso del subdesarrollo y la exclusión.

Nuestro equipo de investigación ha llevado a cabo numerosos estudios de programas de laptops en instituciones educativas, tanto en México (Cervantes, Warschauer, Nardi, y Sambasivan, 2011) como en los Estados Unidos (Warschauer, 2007, 2008; Warschauer, Zheng, Niiya, Cotten, y Farkas, 2014). Estos estudios han confirmado la importancia de prestar atención a cada uno de estos cuatro grupos de recursos para así lograr una mejora educativa significativa con medios digitales.

\section{Conclusión}

A medida que la preocupación sobre las habilidades del siglo XXI es mayor, los esfuerzos globales se han encaminado al incremento del acceso a las TIC y la educación. Sin embargo, el replanteamiento sobre la desigualdad en el acceso a las TIC en términos de una brecha binaria es problemático. El uso de la literacidad como una analogía para el uso de las TIC puede resultar en un marco más comprensivo y productivo para la brecha digital.

A ello se debe agregar que desafiar la noción de la gran brecha digital y desarrollar una comprensión más sofisticada de la literacidad no hizo que la importancia de la literacidad disminuyera. Por el contrario, con una 
mejor comprensión de la literacidad, los educadores y los que formulaban las políticas pudieron promoverla mejor. Aquellos que han criticado los marcos de literacidad asociales y simplistas han tomado la delantera de los esfuerzos para extender la literacidad, empezando por Paulo Freire, quien no solo fomentó el concepto crítico fundamentalmente social de la literacidad, sino que también ayudó a elaborar campañas de alfabetización masiva en varios países del mundo.

Asimismo, una crítica de la noción de la brecha digital es necesaria para informar a cabalidad y desencadenar esfuerzos en cuanto al uso de tecnologías en la promoción de la inclusión social. Las nociones demasiado simplistas de la brecha digital conducen a todo tipo de resultados problemáticos mostrados en las viñetas anteriores; $y$, a su vez, alimentan a los que les gustaría poner fin al financiamiento tecnológico de las comunidades.

Ante este panorama, un marco tecnológico para la inclusión social nos permite reorientar el enfoque de los vacíos que se superan con la entrega de equipos hacia el desarrollo social que se mejora a través de la integración efectiva de las TIC en las comunidades e instituciones. Esta clase de integración solo puede ser alcanzada con la consideración de una amplia gama de recursos físicos, digitales, humanos y sociales que implica el acceso significativo a las TIC.

Aquellos que popularizaron el término «brecha digital» han ayudado a enfocar la atención del público en el importante tema social de la tecnología y la desigualdad. Un nuevo marco puede contribuir a aclarar el rol del uso de las TIC como medio para -y producto de- la inclusión social. Ahora, es momento de profundizar este tema en el entendimiento público a través de una evaluación más completa de lo que significa acceder a las TIC y el propósito de tal acceso.

\section{Agradecimientos}

El presente artículo emana de la discusión anterior del primer autor de estos temas encontrados en Warschauer (2002).

\section{Notas biográficas}

Mark Warschauer es profesor y Decano de Educación en la Universidad de California en Irvine, donde dirige el Laboratorio de Aprendizaje Digital. Es autor o editor de más de 10 libros y de 150 artículos, que incluyen «Learning in the Cloud: How (and Why) to Transform Schools with Digital Media» (Teachers College Press). Fue el editor fundador de la revista Language Learning \& Technology y es el editor que ha inaugurado la nueva revista AERA Open. Su investigación en medios digitales y aprendizaje ha sido financiada por la National Science Foundation, Google Research, la Spencer Foundation, y la Haynes 28 I Foundation, entre otras entidades. 
Melissa Nirya es estudiante doctoral en la Escuela de Educación de la Universidad de California en Irvine. Previamente, ha sido voluntaria en la Little Tokyo Service Center en Los Angeles, organización sin fines de lucro, donde dictó cursos sobre literacidad digital y medios y tecnología a jóvenes y adultos marginales. También, ha trabajado en una universidad tecnológica para desarrollar aplicaciones de aprendizaje virtuales para la educación en salud. Su investigación aborda las literacidades digitales, la actividad de los medios sociales y la ecología del uso de la tecnología por parte de los estudiantes tanto dentro como fuera de la escuela.

\section{Referencias}

Aichholzer, G., y Schmutzer, R. (2001). The digital divide in Austria. Vienna: Institute of Technology Assessment, Austrian Academy of Sciences.

Arora, P. (2010). Hope-in-the-Wall? A digital promise for free learning. British Journal of Educational Technology, 41(5), 689-702.

Becerra, O. (2010). What is reasonable to expect from information and communication technologies in education? Educational Technology Debate. Recuperado de http://edutechdebate.org/computer-configurations-for-learning/

Beuermann, D. W., Cristia, J. P., Cruz-Aguayo, Y., Cueto, S., y Malamud, O. (2013, febrero). Home computers and child outcomes: Short-term impacts from a randomized experiment in Peru (NBER Working Paper No. 18818). Cambridge, MA: National Bureau of Economic Research.

Breitkopf, A. (2012). Cultural and educational implications of global media. The One Laptop per Child Initiative in rural Peruvian schools (Tesis de maestría). University of Hamburg, Hamburg, Germany. Recuperado de http://www.antje-breitkopf.com/wp-content/uploads/2013/01/OLPC_ thesis2012.pdf

Brown, D. C. (1980). Electricity for rural America: The fight for the REA. Westport, Connecticut: Greenwood Press.

Carvin, A. (2000). Mind the gap: The digital divide as the civil rights issue of the new millennium. Multimedia Schools, 7(1), 56-58.

Castells, M. (1997). The power of identity. Malden, MA: Blackwell. (1998). End of Millennium. Malden, MA: Blackwell.

Cervantes, R., Warschauer, M., Nardi, B. y Sambasivan, N. (2011, mayo). Infrastructures for low-cost laptop use in Mexican schools. Proceedings of the SIGCHI. Conference on Human Factors in Computing Systems (pp. 945-954). Recuperado de http://dl.acm.org/citation.cfm?doid=1978942.1979082

Charbonnier, G. (1973). «Primitive» and «civilized» peoples: A conversation with Claude Lèvi-Strauss. The Future of Literacy. Englewood Cliffs, NJ: Prentice-Hall. 
Cisler, S. (2000). Subtract the digital divide (Online essay). Recuperado de http://home.inreach.com/cisler/divide.htm

Cristia, J., Ibarrarán, P., Cueto, S., Santiago, A. y Severín, E. (2012). Technology and Child Development: Evidence from the One Laptop per Child Program. Washington D.C.: Inter-American Development Bank. Recuperado de http://idbdocs.iadb.org/wsdocs/getdocument.aspx?docnum=36706954

De Castell, S. y Luke, A. (1986). Models of literacy in North American schools: Social and historical conditions and consequences. En De Castell, S., Luke, A. y Egan, K. (Eds.). Literacy, society, and schooling: A reader. Cambridge, MA: Cambridge University Press.

DeBoer, J. (2009). The relationship between environmental factors and usage behaviors at «Hole-in-the-wall» computers. International Journal of Educational Development, 29(1), 91-98.

Derndorfer, C. (2010, agosto). One Laptop per Child in South America: Reports from on-the-ground [Speech given at the World Bank, Washington DC]. Ustream. Recuperado de http://www.ustream.tv/recorded/9251465

(2012). Una entrevista con Sandro Marcone acerca de Una Laptop por Niño en el Perú. OLPC News. Recuperado de http://www.olpcnews. com/countries/peru/una_entrevista_con_sandro_marcone_acerca_de_ una_laptop_por_nino.html

DiMaggio, P., Hargittai, E., Celeste, C. y Shafer, S. (2004). Digital inequality: From unequal access to differentiated use. Social inequality, 355-400.

Eisenstein, E. L. (1980). The printing press as an agent of change. Cambridge, MA: Cambridge University Press.

England, L. (2007). Technology applications in English language teaching in Egyptian universities: A developing relationship. Calico Journal, 24(2), 381-406.

Freire, P. (2000). Pedagogy of the oppressed. New York: Continuum.

Freire, P. y Macedo, D. (2013). Literacy: Reading the word and the world. Nueva York: Routledge.

Gee, J. P. (2007). Social linguistics and literacies. Nueva York: Routledge.

Goody, J. (Ed.). (1975). Literacy in traditional societies. Cambridge, MA: Cambridge University Press.

Goody, J. y Watt, I. (1963). The consequences of literacy. Comparative studies in society and history, 5(03), 304-345.

Greenfield, P. M. (1972). Oral or written language: The consequences for cognitive development in Africa, the United States and England. Language and Speech, 15(2), 169-178.

Grimes, D. y Warschauer, M. (2008). Learning with laptops: A multi-method case study. Journal of Educational Computing Research, 38(3), 305-332. 
Gush, K. y de Villiers, R. (2010, Octubre). Application usage of unsupervised digital doorway computer kiosks in remote locations in South Africa. En Proceedings of the 2010 Annual Research Conference of the South African Institute of Computer Scientists and Information Technologists (pp. 93103). Recuperado de http://dl.acm.org/citation.cfm?id=1899514

Hargittai, E. (2002). Second-level digital divide: Differences in people's online skills. First monday, 7(4).

Hargittai, E. y Walejko, G. (2008). The Participation Divide: Content creation and sharing in the digital age 1. Information, Community and Society, 11(2), 239-256.

Ireland Department of Communications, Energy, and Natural Resources (2013). Doing more with digital: National digital strategy for Ireland: Phase 1 - digital engagement. Recuperado de http://www.dcenr.gov.ie/NR/ rdonlyres/54AF1E6E-1A0D-413F-8CEB-2442C03E09BD/0/NationalDigitalStrategyforIreland.pdf

Jarboe, K.P. (2001). Inclusion in the information age: Reframing the debate. Athena Alliance. Recuperado de http://www.athenaalliance.org/inclusion. html

Lievrouw, L. A. (2000). The information environment and universal service. The information society, 16(2), 155-159.

Livingstone, S. y Helsper, E. (2007). Gradations in digital inclusion: children, young people and the digital divide. New media \& society, 9(4), 671-696.

Luria, A.R. (1976). Cognitive development: Its cultural and social foundations. Cambridge, MA: Harvard University Press.

McLuhan, M. (2011). The Gutenberg galaxy: The making of typographic man. Toronto: University of Toronto Press.

McQuillan, H. (2002). Ennis information age town: virtuality rooted in reality. Digital Cities II: Computational and Sociological Approaches (pp. 139-151). Berlin: Springer.

Mitra, S. y Dangwal, R. (2010). Limits to self-organising systems of learningthe Kalikuppam experiment. British Journal of Educational Technology, 41(5), 672-688.

Mooney, John (2001). Comunicación personal. University College Dublin, mayo 2001.

National Telecommunications and Information Administration (2011). Exploring the digital nation: Computer and Internet use at home. Washington, DC: NTIA.

Olson, D. R. (1977). From utterance to text: The bias of language in speech and writing. Harvard educational review, 47(3), 257-281.

Patterson, R. y Wilson III, E.J. (2000). New IT and social inequality: Resetting the research and policy agenda. The Information Society, 16(1), 77-86. 
Salinas, A. y Sánchez, J. (2009). Digital inclusion in Chile: Internet in rural schools. International Journal of Educational Development, 29(6), 573582.

Santiago, A., Severin, E., Cristia, J., Ibarrarán, P., Thompson, J. y Cueto, S. (2010). Evaluación experimental del programa «Una Laptop por Niño» en Perú. Washington, DC: Banco Interamericano de Desarrollo.

Scribner, S. y Cole, M. (1981). The psychology of literacy (Vol. 198, No. 1). Cambridge, MA: Harvard University Press.

Selwyn, N. (2004). Reconsidering political and popular understandings of the digital divide. New Media \& Society, 6(3), 341-362.

Stewart, A. (2000). Social inclusion: An introduction. En P. Askonas y A. Stewart (Eds.), Social inclusion: Possiblities and tensions (pp. 1-16). Londres: Macmillan.

Street, B.V. (1995). Social literacies: Critical approaches to literacy in development, ethnography and education (Vol. 1995). Londres: Longman.

Warschauer, M. (2002). Reconceptualizing the digital divide. First Monday, 7 (7). Recuperado de http://firstmonday.org/article/view/967/888.

(2003a). The allures and illusions of modernity: Technology and educational reform in Egypt. Education Policy Analysis Archives, 11(38).

(2003b). Technology and social inclusion: Rethinking the digital divide. Cambridge, MA: MIT Press.

(2004). The rhetoric and reality of aid: Promoting educational technology in Egypt. Globalisation, Societies and Education, 2(3), 377390 .

(2007). Information literacy in the laptop classroom. Teachers College Record, 109(11), 2511-2540.

(2008). Laptops and literacy: A multi-site case study. Pedagogies, 3(1), 52-67.

Warschauer, M. y Ames, M. (2010). Can One Laptop per Child Save the World's Poor? Journal of International Affairs, 64(1), 33-51.

Warschauer, M. y Matuchniak, T. (2010). New technology and digital worlds: Analyzing evidence of equity in access, use, and outcomes. Review of Research in Education, 34(1), 179-225.

Warschauer, M., Zheng, B., Niiya, M., Cotten, S. y Farkas, G. (2014). Balancing the one-to-one equation: Equity and access in three laptop programs. Equity \& Excellence in Education, 47(1), 46-62. 